\title{
Untersuchung der Nebeldecke mit Hilfe von ERTS-1-Bildern
}

\section{ERTS und Meteorologie}

ERTS-1 ist nicht ein meteorologischer Satellit. Die raumzeitliche Bedeckung ist zu spärlich, und Aufnahmen werden in der Regel nur bei geringer Bewölkung gemacht.

Dessen ungeachtet können ERTS-Bilder als wertvolle Zusatzinformation für meteorologische Einzelfallanalysen beigezogen werden. Die gute Bildauflösung über ein vergleichsweise sehr großes Gebiet gestattet es, kleinräumige Effekte in einen größeren Zusammenhang zu stellen.

In einer ersten Untersuchung befaßte sich PIAGET (1973) mit Fragen der Bewölkungsinterpretation im Alpenraum. Dabei konnten Prozesse der Bewölkungsauflösung über Tälern und die Entwicklung von parallel angeordneten Wolkenstraßen in Abhängigkeit des Reliefs untersucht werden.

Besonders eindrücklich präsentiert sich auf mehreren Aufnahmen (20./21. 9. 1972 und 18./19./20. 12. 1972) die Nebelverbreitung, wobei Nebelmeerobergrenze und dynamische Strukturen ins Auge springen. An anderer Stelle konnte auf den räumlich komplexen Aufbau der Nebeldecke durch Kombination von Satellitenbild und terrestrischer Beobachtung hingewiesen werden (WINIGER, 1974). Im folgenden sollen am Beispiel der Aufnahme E-1149-09502/4-6 vom 19.12.1972 9.50 GMT einige weitere Auswertungsmöglichkeiten diskutiert werden.

\section{Nebelausdehnung und -obergrenze}

Mit der Nebelmeerobergrenze wird ein ungefähres Inversionsniveau festgelegt und damit theoretisch die räumliche Ausdehnung der Kaltluftmasse. Alle bis jetzt vorliegenden Nebelaufnahmen belegen den überragenden Einfluß des Reliefs, der im hier diskutierten Beispiel durch die kräftige Luftströmung noch gesteigert wird. In Anlehnung an die Großgliederung der Landschaft in Jura, höheres und tieferes Mittelland und Alpen lassen sich Nebelregionen mit vergleichsweise ähnlichem Aufbau und Verhalten der Nebeldecke ausscheiden:

1. Der Raum nördlich Basels mit einer in mindestens zwei Stockwerke gegliederten Nebelschicht (höhere Schicht ca. $700 \mathrm{~m}$ über der tieferen; Berechnung aus Schattenwurf) mit lokalen Auflösungstendenzen über dem Schwarzwald (1).
2. Mittelland: Der ausgesprochene Kanalisierungseffekt der Luftströmung gegen SW ist klar erkennbar. Die Nebelobergrenze kann auf \pm 50 m genau festgestellt werden, v. a. im Randbereich und an einzelnen «Nunatakern». Sie beträgt im Raum Hörnli-Kreuzegg (2) und am Gibloux (3) 1100-1200 m, steigt an der Luvseite exponierter Bergzüge auf 1200-1400 m [Napf (4), Weißenstein (5)] und ist auf der Leeseite entsprechend tiefer anzusetzen oder fehlt überhaupt (Napf).

3. Alpentäler: Diese sind je nach Richtung mit teilweise stagnierenden Nebelmassen angefüllt. Mit Ausnahme der nach Norden geöffneten Täler [Glarner Gebiet (6), Uri (7)] sind meist nur die untersten Talabschnitte bedeckt, wobei die Nebelgrenze wesentlich tiefer als im Mittelland liegt [Frutigen (8), Sierre (9)]. Neben der Orographie dürften lokale Windsysteme für diese Verteilung verantwortlich gemacht werden.

4. Die nebelfreien Bereiche des Juras, Emmentals und der Alpen: Einesteils ragen sie über das Nebelmeer hinaus, häufig aber sind sie von der Zufuhr der notwendigen Kaltluft abgeschnitten [z. B. Simmental (10)].

\section{Nebeldichte}

Im allgemein gut abgrenzbaren Nebelmeer treten vereinzelt auch über Tälern Löcher und Stellen mit nurmehr schwacher Dunstbedeckung auf [z. B. Aaretal südlich Bern (11)]. Wieweit hier unterschiedliche thermische Verhältnisse des Untergrundes (Seen, Städte usw.) wirksam werden, müßte abgeklärt werden.

\section{Strukturen}

Wo das Wettersatellitenbild (ESSA, NOAA) der geringen Auflösung wegen ein weitgehend ruhig daliegendes Nebelmeer vortäuscht, zeigt das ERTS-Bild ein verwirrend vielfältiges Bild von Oberflächenstrukturen, die sich in Flächen, Linien und Punkte einteilen lassen.

(1-20) Diese Zahlen beziehen sich auf die in der Abbildung eingesetzten Nummern.

Dr. M. Winiger, Geographisches Institut der Universität Bern, Hallerstraße 12, 3012 Bern. 


\section{Flächen}

Das Bild bestätigt, daß ein Nebelmeer laminar aufgebaut ist. Schuppenförmig lagern mehrere Nebelpakete übereinander oder werden übereinandergeschoben. Dieser Effekt ist kleinräumig zu erkennen [über dem Thuner See (12)] oder es lassen sich Grenzlinien über große Distanzen verfolgen [am Südrand des Schwarzwaldes (13)].

\section{Wellen}

Die ganze Nebeloberfläche ist von einem dichten Netz von Wellenlinien überzogen, die sich bezüglich Richtung, Abstand der Wellenzüge und Dämpfung unterscheiden und die an vielen Stellen interferieren (etwa östlich des Weißensteins). Sie können in zwei genetisch unterschiedliche Gruppen eingeteilt werden: 1. Hinderniswellen und 2. Kanalisierungswellen in Tälern. Es wäre zu prüfen, ob die von BOUET und KUHN (1970) beschriebenen Gravitationswellen ebenfalls nachgewiesen werden könnten.

Bei den Hinderniswellen veranschaulicht die Nebeloberfläche die Bewegung des gesamten Luftmassenpaketes. Deutlich lassen sich dabei zwei Fälle unterscheiden. 1. Das Hindernis ragt über die Nebelgrenze hinaus: es lösen sich keilförmige Wellenzüge (Hörnli, Napf, Jura) ähnlich der Spur eines Schiffes. 2. Der Nebel überströmt den Höhenrücken: Die Wellen lösen sich wie die bekannten Leewellen einer Föhnlage parallel zum Hindernis ab (über dem Schwarzwald). Die Kanalisierungswellen dürften als Grenzflächenphänomen zwischen zwei unterschiedlich bewegten Luftmassen zu deuten sein. In unserem Fall überstreicht ein Bergwind die taleinwärts drängende Kaltluft. Dieser übergelagerte Wind verliert seinen Einfluß erst über dem sich verbreiternden Vorland [z. B. Walliser Wind über dem Genfer See (14)].

\section{Punktmuster}

Durch lokale Thermik kann sich die Nebeldecke punktförmig aufwölben. Bekannt sind die Nebelpilze über einzelnen Hochkaminen. Auch im Satellitenbild zeichnen sich die Industrie-Standorte von Cressier (15) und Aigle (16) ab, doch werden die Aufwölbungen durch die vorbeiziehenden Wellenzüge überlagert.

\section{Windfeld}

Anordnung und Ausprägung der Wellenzüge veranschaulichen letzten Endes das Windfeld und seine nachhaltige Beeinflussung durch die Topographie. Nach Angabe der Wetterkarte herrschten in der Höhe NE-Winde, am Boden E-Winde vor. Das Schweizerische Mittelland befindet sich auf der Rückseite eines Hochdruckgebietes, das sich langsam ostwärts entfernt. Im tieferen Mittelland wird diese großräumige Strömung kanalisiert und durch die allmähliche Einschnürung gegen Westen hin eher noch verstärkt, während sie im quer dazu stehenden Aaretal fast völlig unterbunden wird.

Fallwinde am Brünig (17) und am Genfer See (18) führen zu lokalen Nebelauflösungen. Die Luftströmungen aus zahlreichen Tälern folgen wie Wasser einem Stromstrich und verlieren sich in Deltazonen [Justistal-Thuner See (19)]. Am Genfer See [über Montreux (20)] konvergieren Winde aus verschiedenen Richtungen. Der Katalog lokaler Phänomene ließe sich beliebig weiterführen.

Es wäre zweifellos lohnend, die genauen Windrichtungen und aus den Wellenlängen, bzw. den Frontwellenwinkeln die zugehörigen Geschwindigkeiten zu berechnen. Das Windfeld ließe sich kaum auf andere Weise derart gut bestimmen, wobei einschränkend natürlich festgehalten werden muß, daß dies nur gerade im Niveau der Nebeloberfläche mit genügender Genauigkeit möglich sein dürfte.

\section{Zusammenfassung}

Zusammenfassend darf festgehalten werden, daß ERTSAufnahmen als zusätzliches Informationsmaterial für Einzelfallanalysen berücksichtigt werden können. Sie vermitteln Ideen über Zusammenhänge zwischen Kaltluftverbreitung und Strömungsdynamik als Funktion einer Großwetterlage und des lokalen Reliefs. Dies wohlverstanden, am individuell ausgeprägten Einzel-

Nebelverbreitung über dem Schweizerischen Mittelland am 19. Dezember 1972, 9.50 Uhr GMT (Goddard Space Flight Center, Greenbelt, Maryland, E-1149-09502/4-6). Die Nummern beziehen sich auf die im Text diskutierten Geländestellen. 

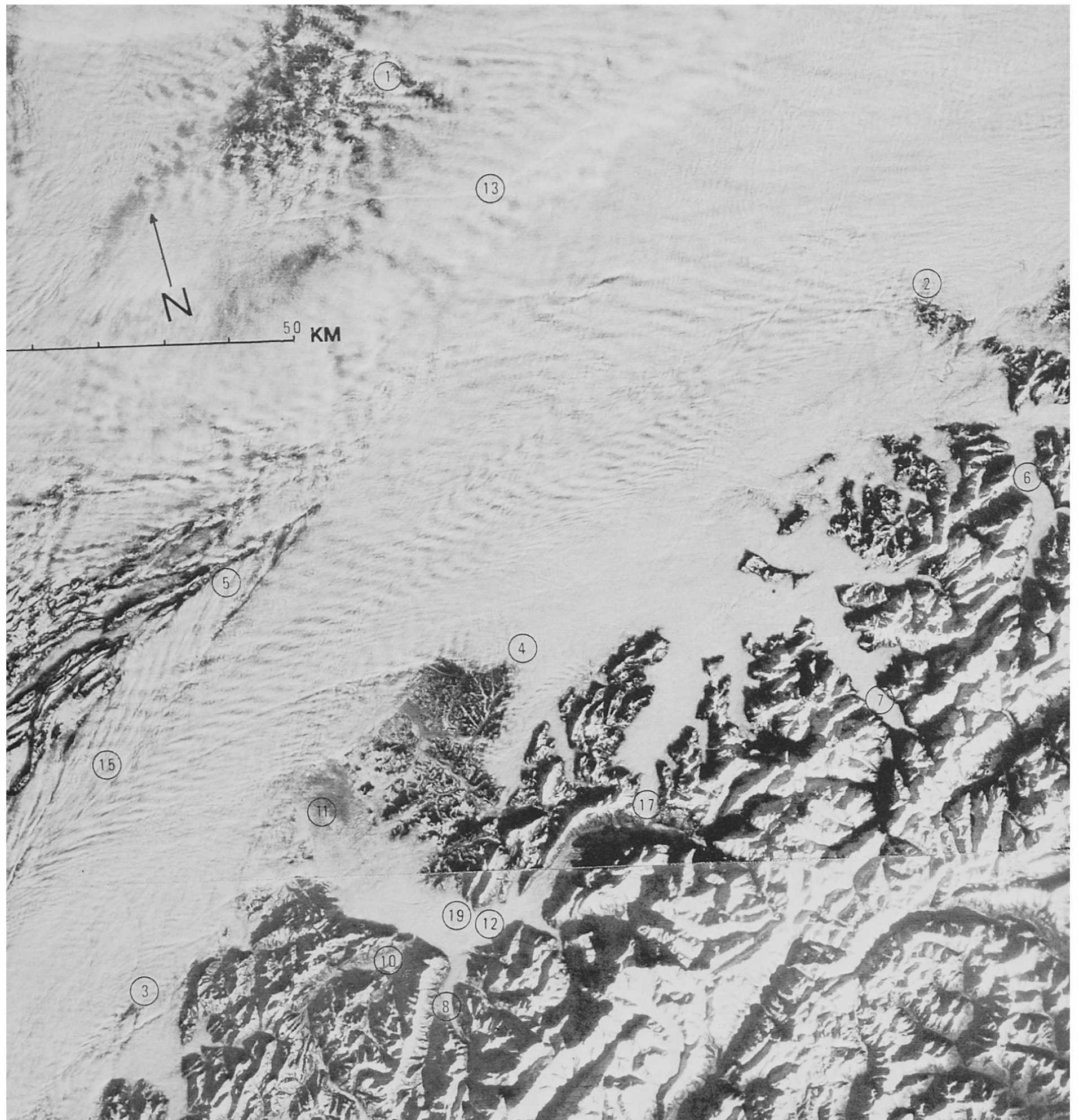

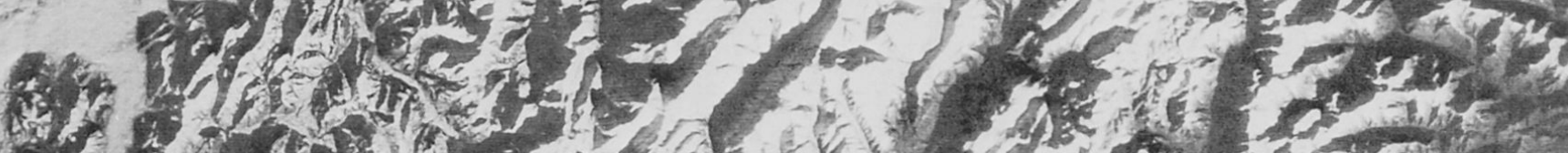

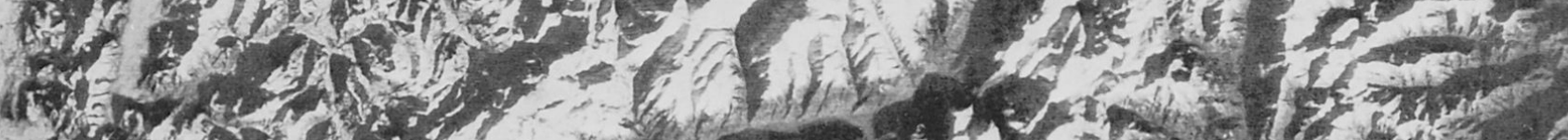

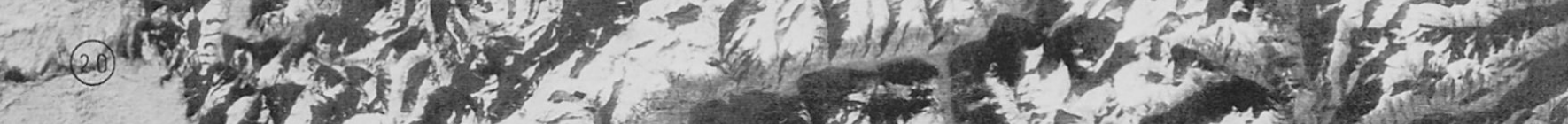

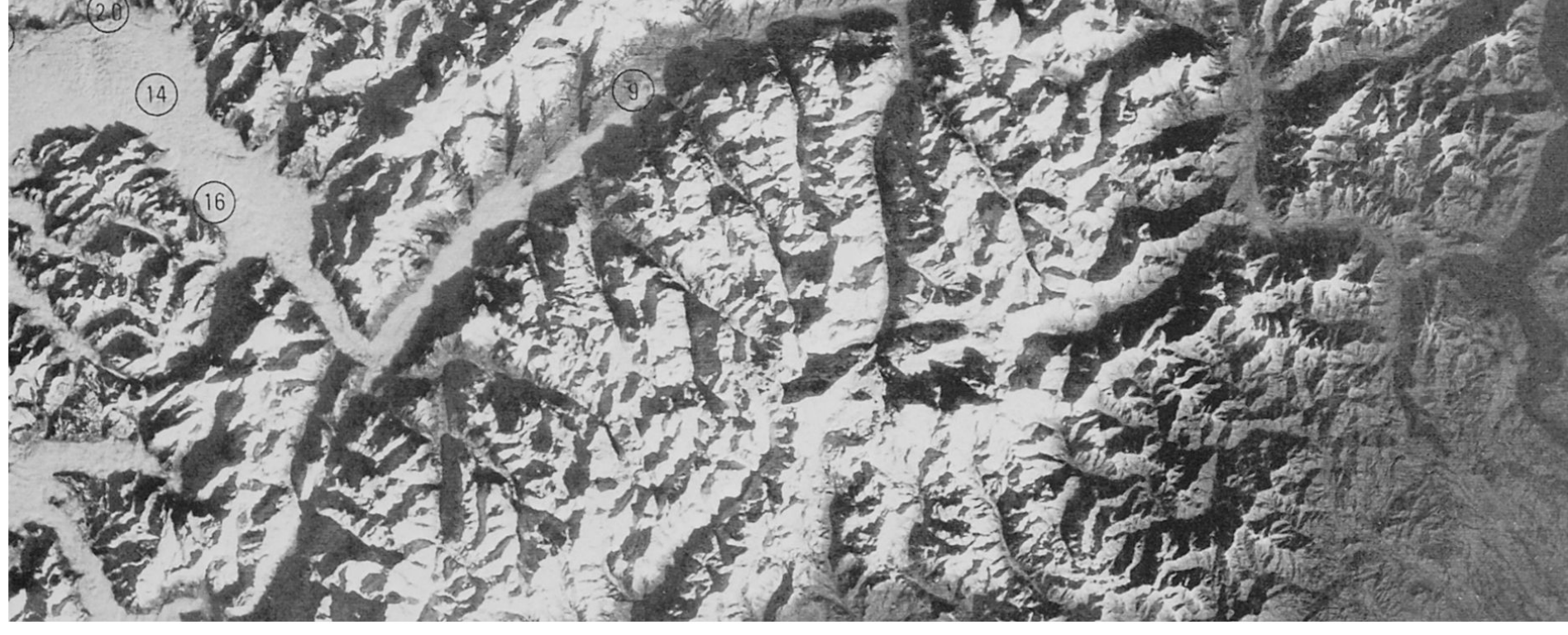


ereignis. Für eine systematische Klassifizierung sind wir nach wie vor auf die terrestrischen Beobachtungen, Sondenaufstiege und Wettersatellitenaufnahmen (SCHACHER, 1974) angewiesen. Doch lassen die geplanten Projekte (etwa Meteosat) einen ausgeprägten Trend zum Beizug von Fernerkundungsdaten in noch weitergehendem Maße als heute als wahrscheinlich annehmen.

\section{Literatur}

BOUET, M. Und KUHN, W., 1970: Vagues de brouillard considérées comme ondes de gravité. Verh. Schweiz. Nat. f. Ges., $150: 170-172$.

PIAGET, A., 1973: First preliminary report on meteorological interpretation of clouds or cloud systems appearing on pictures of the Alpine region received from the Earth's Resources Satellite ERTS-1. Arbeitsberichte der Schweiz. MZA, No. 37, Zürich.

SCHACHER, F., 1974: Nebelkarte der Schweiz. Unpubl. Diplomarbeit, Geogr. Institut Univ. Zürich.

wINIGER, M., 1974: Die raum-zeitliche Dynamik der Nebeldecke aus Boden- und Satellitenbeobachtungen.

Inform. u. Beitr. z. Klimaforschung; $12: 24-30$. 